\title{
A Fusion Location Algorithm Based on Federated Kalman Filtering
}

\author{
LV Long-huil, a , JIN Hong-xia ${ }^{1, b}$ \\ ${ }^{1}$ Henan Quality Engineering Vocational College, Pingdingshan HeNan 467001, China \\ azcysym@126.com, bdjxzcy@126.com
}

Keywords: Federal Kalman Filter; WLAN System; RFID System; Location Algorithm; Fusion Algorithm

\begin{abstract}
The paper designed a WLAN system with RFID systems joint locating algorithm based on Federated Kalman Filtering. For the problem in indoor mobile node single positioning system, such as insufficient positioning accuracy, longer positioning time and not ideal algorithm complexity, it was proposed that the federated filtering techniques was applied in the location system, and was combined with data from WLAN and RFID to realize all accuracy improved. In the system, sub-filter use unscented Kalman filter algorithm independent measurement updates and time update, and the subsystem information is fused with the main filter based on no feedback mode. It was proved from the result that the calculation improved was of feasibility, which reduced unscented Kalman filter calculation, decreased maximum deviation and minimum deviation and improved the positioning accuracy.
\end{abstract}

\section{Introduction}

With the increase of the number of mobile devices and improvement of the degree of intelligence, location technology [1] is highly valued by the researcher as the core support functions of various kinds of mobile applications. At present, common indoor positioning technology includes ultrasonic positioning technology, WLAN positioning technology, Zigbee positioning technology, infrared positioning technology and RFID positioning technology. One single positioning technology is difficult to achieve a satisfactory result in practice, so multi-sensor data fusion technology has become an important issue in the field of indoor mobile node localization.

WLAN positioning technology has developed rapidly in recent years, which mainly includes Nibble system[2] based on acquisition and analysis of noise ratio or signal quality, WHAM! Positioning system[3] based on receiving the signal strength of AP, Ekahau positioning system[4] , Horus positioning systems and Rice systems.The RFID system,whose positioning time is shorter, the precision is higher, received much concern for its advantages of non-line and non contract ,although the positioning range is limited to the distance between the transmitter and the receiver .This paper is to effectively improve the positioning accuracy of the mobile node and real-time through information fusion of WLAN positioning system and RFID positioning system based on Federated Kalman Filtering, which has certain practical value.

\section{Theoretical basis}

\section{Federated Kalman Filtering Model}

Traditional Kalman filtering technology for multi-sensor data fusion methods generally includes centralized Kalman filtering and the decentralized Kalman filter. Federated filter proposed by Carlson[5] achieves good effect in practical application because of its small amount of calculation, good fault-tolerant performance and flexible design.

Fusion algorithm based on Federated Kalman Filtering includes algorithm both whose estimate for each sub filter is correlated and uncorrelated. Due to the premise that the various sub filters is uncorrelated can not be guaranteed in the actual use,this paper mainly study the fusion algorithm for the estimate of each sub filter,using the variance upper bound technique to transform the filtering algorithm,thus each sub filter will be estimated from the correlated state.Federated filter is a kind of two-stage filtering structure. It is assumed that the state estimation of each sub filter can be 
expressed as: $\hat{X}_{i}=\left[\begin{array}{l}\hat{X}_{c i} \\ \hat{X}_{b i}\end{array}\right]$. $\hat{X}_{c i}$ is the estimate of the common state $X_{c}$ for each sub filter. $\hat{X}_{b i}$ is the proprietary state estimate of filter i .

\section{Process design of Federated Kalman filter}

Under normal circumstances, federated filter process mainly includes four processes as follow : information distribution, information update, measurement update of information and information fusion.

Step1 Distribution of information.Determine the proportionality coefficient of information distribution,between the main filters and sub filters as shown in formula (1). $Q_{k}^{-1}$ and $\left(P_{k \mid k}^{g}\right)^{-1}$ refer to system process information.In formula (1), parameter $\beta_{i}$ is called an information distribution coefficient to meet the conditions of $\beta_{i}>0$ and distribution principle in formula (2).

$$
\left\{\begin{array}{l}
Q_{k}^{i}=\beta_{i}^{-1} Q_{k} \\
P_{k \mid k}^{i}=\beta_{i}^{-1} P_{k \mid k}^{i} \\
\hat{X}_{k \mid k}^{i}=\hat{X}_{k \mid k}^{g}, i=1,2, \ldots, N, m
\end{array}\right.
$$

$$
\sum_{i=1}^{N} \beta_{i}+\beta_{m}=1
$$

Step 2 Time update of information. The process goes respectively between the senior filter and sub filters independently by means of the filtering algorithm shown in the formula (3).

Step 3 Measurement update of information. The update is carried out in the sub filters of the system, while the main filter does not need to carry on this step. The measurement update algorithm for each sub filter is shown in formula (4).

$$
\left\{\begin{array}{l}
\hat{X}_{k+1 \mid k}^{i}=\Phi_{k} \hat{X}_{k \mid k}^{i} \\
P_{k+1 \mid k}^{i}=\Phi_{k} P_{k \mid k}^{i} \Phi_{k}^{T}+\Gamma_{k} Q_{k}^{i} \Gamma_{k}^{T}, i=1,2, \ldots, N, m
\end{array}\right.
$$

$$
\left\{\begin{array}{l}
\left(P_{k+1 \mid k+1}^{i} i\right)^{-1}=\left(P_{k+1 \mid k}\right)^{-1}+\left(H_{k+1}^{i}\right)^{T}\left(R_{k+1}^{i}\right)^{-1} H_{k+1}^{i} \\
\left(P_{k+1 \mid k+1}^{i}\right)^{-1} \hat{X}_{k+1 k+1}^{i}=\left(P_{k+1 k}^{i}\right)^{-1} \hat{X}_{k+1 \mid k}^{i}+\left(H_{k+1}^{i}\right)^{T}\left(R_{k+1}^{i}\right)^{-1} Z_{k+1}^{i} \\
, i=1,2, \ldots, N
\end{array}\right.
$$

Step 4 Fusion of information. The local estimation information of each subsystem will be fused by the given algorithm formula to get the optimal global estimation. It is the core part of the federated filter, and the fusion algorithm is shown in the formula (5) and (6).

$$
\begin{aligned}
& \hat{X}_{k+1 \mid k+1}^{g}=P_{k+1 \mid k+1}^{g}\left[\left(P_{k+1 \mid k+1}^{1}\right)^{-1} \hat{X}_{k+1 \mid k+1}^{1}+\left(P_{k+1 \mid k+1}^{2}\right)^{-1} \hat{X}_{k+1 \mid k+1}^{2}\right] \\
& P_{k+1 k+1}^{g}=\left[\left(P_{k+1 k+1}^{1}\right)^{-1}+\left(P_{k+1 k+1}^{2}\right)^{-1}+\ldots+\left(P_{k+1 k+1}^{N}\right)^{-1}+\left(P_{k+1 \mid k+1}^{m}\right)^{-1}\right]_{(5)}^{-1}+P_{k+1 k+1}^{g}\left[:: *+\left(P_{k+1 k+1}^{N}\right)^{-1} \hat{X}_{k+1 \mid k+1}^{N}+\left(P_{k+1 k+1}^{m}\right)^{-1} \hat{X}_{k+1 k k+1}^{m}\right]
\end{aligned}
$$

Through the above four steps of process design, optimal solution managed to be obtained in the global equation by re synthesis of non-optimality in the process of information fusion,although variance upper bound technique can lead to some local loss of information.

\section{WLAN / RFID Information fusion and location algorithm}

In information fusion algorithm based on the combination of positioning of WLAN system and RFID system,the federated filter consists of a main filter and two subsystems, as shown in Figure 1. $\hat{X}_{1}$ and $\hat{X}_{2}$ are for state estimates of the output of the two sub-filters. ${ }^{P_{1}}$ and $P_{2}$ are for co-variance matrix of the two sub-filters. $\hat{X}_{g}$ and $P_{g}$ are for optimal estimate and co-variance matrix of the federated filter.

The subsystem WLAN and subsystem RFID accomplish independent measurement and time update through unscented Kalman filtering algorithm, with measurement information parallel processed in subsystem. The information outputed by the sub system is fused with the non feedback mode of the main filter,thus an accurate positioning information will be obtained. 


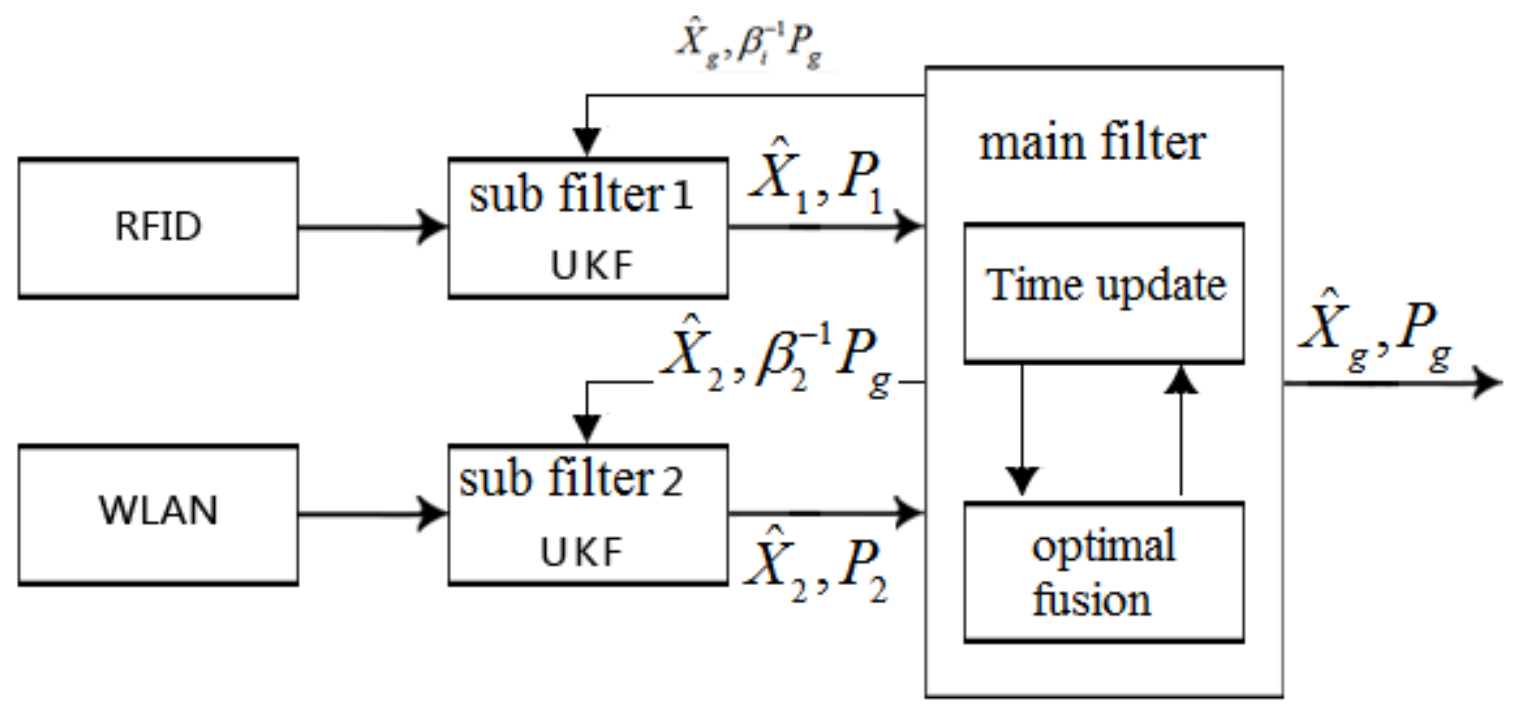

\section{System state equation}

Figure 1 Schematic diagram of RFID/WLAN positioning

The positioning of the mobile node of the system is completed in the two-dimensional plane, so the public state variable $\mathrm{x}$ is taken as follow: $X_{g}=\left[S_{x}, v_{x}, S_{y}, v_{y}\right]^{T} . S_{x}$ and ${ }^{S_{y}}$ show the position of the mobile node in the $\mathrm{X}$ direction and the $\mathrm{Y}$ direction of the vertical coordinate. ${ }^{v_{x}}$ and ${ }^{v_{y}}$ show the velocity in the $\mathrm{X}$ direction and $\mathrm{Y}$ direction of the mobile node. Two sub filters are: ${ }^{X_{1}=\left[X_{g}, W\right]^{T}}$, $X_{2}=\left[X_{g}, V\right]^{T}$. W is for WLAN system error. V is for RFID system error.

\section{System observation equation}

The observation equations of the sub filters are expressed as:

$$
\begin{aligned}
& Z_{1}(k)=H_{1}(k) X_{1}(k)+W_{1}(k), \quad Z_{2}(k)=H_{2}(k) X_{2}(k)+W_{2}(k) \text {.In the equation, } Z_{1}=\left[s_{x}, s_{y}\right]^{T}, \\
& H_{1}(k)=\left[\begin{array}{llllllll}
1 & 0 & 0 & 0 & 0 & 0 & 0 & 0 \\
0 & 1 & 0 & 0 & 0 & 0 & 0 & 0
\end{array}\right], W_{1}(k)=\left[w_{x}(k), w_{y}(k)\right]^{T} .
\end{aligned}
$$

\section{Information fusion of WLAN and RFID}

WLAN system and RFID system have no influence to each other because of their independent work. As a result,in the first step of information distribution of information fusion algorithm,the common reference system coefficient $\beta_{m}$ is taken as 0 ,that is $P_{m}^{-1}=0$. Rather than filter calculation,only information synthetic of two subsystems are needed and at the same time, the estimated value of the main filter is done as global. The main filter algorithm is expressed as follows:

$$
\hat{X}_{k}=P_{k}\left(P_{1, k}^{-1} \hat{X}_{1, k}+P_{2, k}^{-1} \hat{X}_{2, k}\right) \quad \text { (7) } P_{k}=\left(P_{1, k}^{-1}+P_{2, k}^{-1}\right)
$$

In the calculation, take $\beta_{1}=P_{k} P_{1, k}^{-1}, \quad \beta_{2}=P_{k} P_{2, k}^{-1}$, that is $\hat{X}_{1, k}=\hat{X}_{2, k}=\hat{X}_{k}, P_{1, k}^{-1}=\beta_{1} P_{k}^{-1}, P_{2, k}^{-1}=\beta_{2} P_{k}^{-1}$, $Q_{2, k}^{-1}=\beta_{1} Q_{k}^{-1}, Q_{2, k}^{-1}=\beta_{2} Q_{k}^{-1}$.

In federal filter structure, the information allocation algorithm of sub-filter is as follows: $\left(\hat{X}_{i}\right)_{k}=\left(\hat{X}_{c}\right)_{k}, \quad\left(P_{i}\right)_{k}=1 / \beta_{i}\left(P_{c}\right)_{k}, \quad\left(Q_{i}\right)_{k}=1 / \beta_{i} Q_{k}$.

The fusion algorithm for the implementation of the main filter is:

$$
P_{c}=\left[P_{1}^{-1}+P_{2}^{-1}+\ldots+P_{n}^{-1}+P_{m}^{-1}\right]^{-1} \text { (9) } \hat{X}=P_{c}\left[P_{1}^{-1} \hat{X}_{1}+P_{2}^{-1} \hat{X}_{2}+\ldots+P_{n}^{-1} \hat{X}_{n}+P_{m}^{-1} \hat{X}_{m}\right]
$$

Estimates of the global main filter in information fusion is obtained after the fusion of sub filter state vector estimate. The weight of the main filter output varies with distribution coefficient of the subsystem. Although the weight from the main filter to sub filters vary with $\beta_{i}$, it still take 
advantage of the whole information of each subsystem. Optimality after fusion is entirely unrelated to $\beta_{i}$. This paper verifies this conclusion through experiments, as shown in Table 1 , the distribution coefficient of the WLAN subsystem is $0.9,0.1,0.5$, The partition coefficient of the RFID subsystem is $0.1,0.9,0.5$, and the distribution coefficient of the main filter is 0 . The experimental results show that the information distribution coefficient has little influence on the fusion result of the main filter.

Table 1 Comparison for experimental results contrast

\begin{tabular}{|c|l|c|c|c|}
\hline Number & WLAN & RFID & maximum deviation & mean deviation \\
\hline 1 & 0.9 & 0.1 & 0.95 & 0.60 \\
\hline 2 & 0.1 & 0.9 & 0.9 & 0.52 \\
\hline 3 & 0.5 & 0.5 & 0.82 & 0.58 \\
\hline
\end{tabular}

\section{Analysis of simulation result}

Fusion algorithm simulation experiment was carried out in logistics laboratory, indoor enclosure, $15 \mathrm{~m} \times 10 \mathrm{~m}$, covers an area of about $150 \mathrm{~m}^{2}$.In AGV car carrying the mobile node in the laboratory, three groups of experiments were conducted, the process is as follows:

\section{Test environment}

Three groups of experiments are WLAN system positioning experiment, RFID system positioning experiment and WLAN/RFID joint positioning experiment. WLAN system is composed of three routers, W1, W2, W3, used to maintain the wireless network card of the mobile node in the same plane. RFID system experiment goes with 7 RFID tags ,R1, R1.. R7, with its reader carried by the mobile node.Make cell division in the laboratory within $0.5 \mathrm{~m} * 0.5 \mathrm{~m}$, and establish coordinate system. The coordinates of the router and RFID tags are: W1 $(3.0 \mathrm{~m}, 1.0 \mathrm{~m}), \mathrm{W} 2(1.0 \mathrm{~m}, 4.0 \mathrm{~m}), \mathrm{W} 3$ (5.5m, 6.0m), R1 (2.5m, 0.5m), R2 (0.5 m and $1.5 \mathrm{~m}), \mathrm{R} 3(0.5 \mathrm{~m}, 4.5 \mathrm{~m}), \mathrm{R} 4(2.0 \mathrm{~m}, 6.0 \mathrm{~m})$, R5 (5.0 and 6.0), R6 (5.5m, 4.0m), R7 $(4.0 \mathrm{~m}$ and $3.0 \mathrm{~m})$. While experimenting, a part of the nodes is selected as samples.

Mobile nodes do four period of continuous linear trajectory along the preset path in the two-dimensional plane, starting node $(4 \mathrm{~m}, \mathrm{~m})$, with $0.02 \mathrm{~m} / \mathrm{s}$ move forward for 240 seconds. In the information fusion algorithm, the sampling time interval is 33S, $X_{g}=\left[\begin{array}{llll}0 & 0.001 & 0 & 0.001\end{array}\right]^{T}$ and the distribution coefficient is : $\beta_{1}=\beta_{2}=0.5$.

\section{Analysis of experimental results}

The results of the three groups are shown in Table 2. Fig. 2 is Comprehensive comparison of experimental results. Experimental results show that the federated filtering structure of information fusion system disperse and reduce the amount of computation of the unscented Kalman filter.Compared to single WLAN positioning system or RFID positioning system,the maximum deviation were reduced by $52.72 \%, 42.86 \%$, and minimum deviation were reduced by $71.74 \%$ and $62.86 \%$ with the use of WLAN/RFID joint information fusion localization algorithm,and therefore positioning accuracy is improved significantly.

Table 2 Comparison of experimental results

\begin{tabular}{|c|c|c|c|}
\hline group number & positioning system & maximum deviation & minimum deviation \\
\hline 1 & WLAN & $1.10 \mathrm{~m}$ & $0.46 \mathrm{~m}$ \\
\hline 2 & RFID & $0.91 \mathrm{~m}$ & $0.35 \mathrm{~m}$ \\
\hline 3 & WLAN/RFID & $0.52 \mathrm{~m}$ & $0.13 \mathrm{~m}$ \\
\hline
\end{tabular}




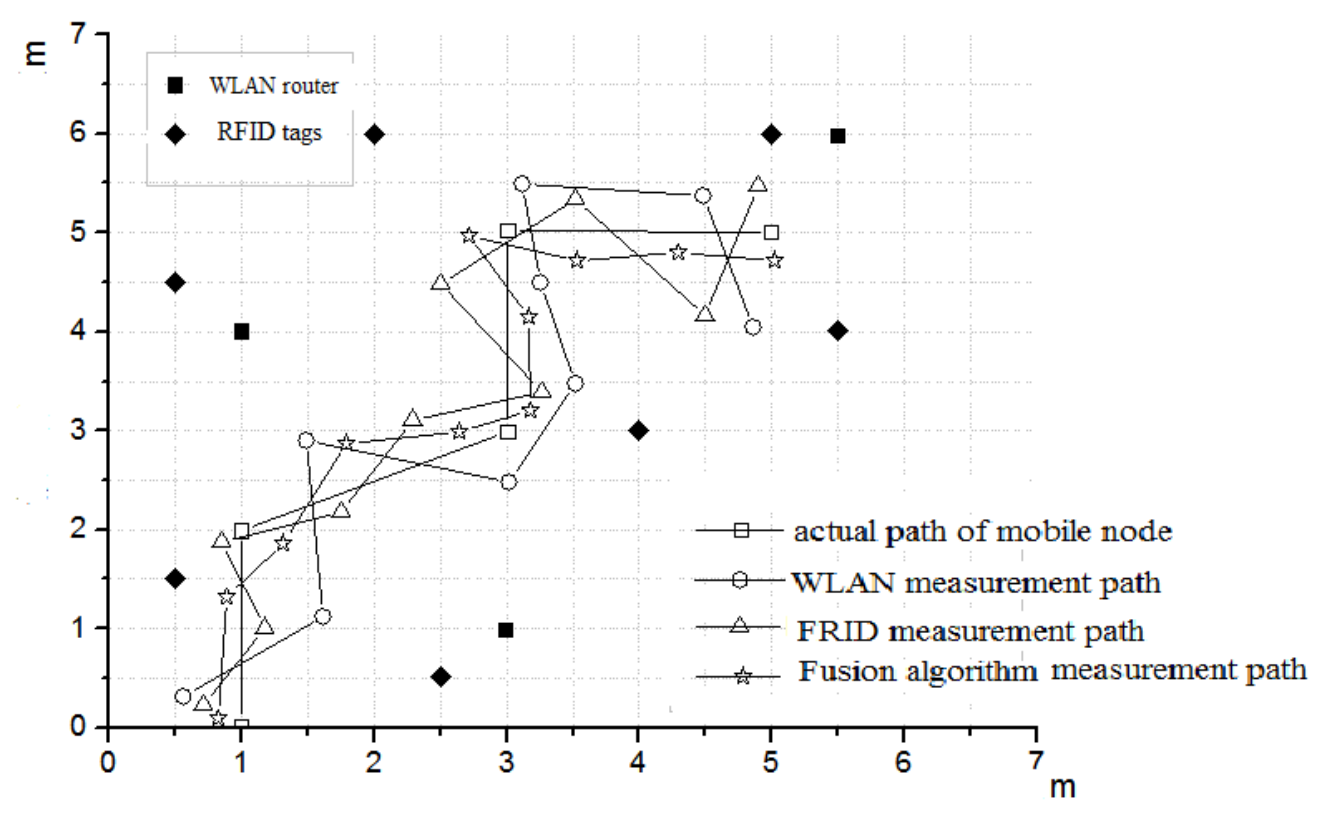

Figure 2 Comparison of experimental results of three groups

\section{Conclusion}

Along with the development of mobile network technology and the enrichment of application of "Internet +”, mobile positioning technology has got some achievement as the core support, although it's still unable to meet various constraints that exist under certain situations or conditions. In this paper, a new information fusion algorithm based on Federated Kalman filtering is designed.This algorithm effectively improves the positioning accuracy and real-time performance of a single positioning system under the premise of not increasing the complexity of the algorithm.

Simulation results show that the proposed algorithm is an efficient location information fusion algorithm in mobile location.

\section{References}

[1] P.Castro,P.Chiu,T.Kremenek,R.Muntz. A probabilistic room location service for wireless networked environments[C].In Proceedings of the 3rd International Conference on Ubiquitous Computing (UbiComp), Georgia, USA, 2001:18-34.

[2] D. L. Lee, Q. Chen. A model-based WiFi localization method[C]. In: Proceedings of Infoscale, 2007:1-7.

[3] S. C. Yeh, Y. Chiou, Y. J. Peng. A research for indoor LBS technology based on RSSI prediction model[J]. Journal of Informatics \& Electronics, 2006, 1(1):1-8.

[4] N. A. Darlson, M. P. Berauducel. Federated kalman filtersimulation results[J]. Navigation, 1994, 41(3):297-321. 